\title{
Predicting Moment Carrying Capacity of the "sagae" Connection Using the Finite Element Method*1
}

\author{
Gi Young Jeong*2†, Moon-Jae Park*3, Joo-Saeng Park*3, and Sang-Joon Lee*3
}

\begin{abstract}
The goal of this study is to analyze the effects of geometries of mortise and tenon on moment carrying capacity of the "sagae" connection. Effects of different tenon widths, mortise depths of connection from the top and bottom beams on stress distribution were investigated using the finite element method (FEM). Critical normal and shear stresses occurred at the reentrant corner from the mortise of the bottom beam. The maximum moment carrying capacity of the sagae connection from the FEM was validated from the results of experimental test. Maximizing moment carrying capacity of the sagae connection was found when the tenon width and mortise depth from the two beams were $40 \mathrm{~mm}$ and $60 \mathrm{~mm}$, respectively.
\end{abstract}

Keywords : sagae connection, finite element method, mortise, tenon

\section{INTRODUCTION}

Various wood to wood connections have been used for traditional wood frame buildings in different countries. Although wood to wood connections have been used for the wood frame buildings, architects and contractors cannot easily access the design value for the wood to wood connections for modern buildings due to the lack of design information for the connections. Modern buildings require the design values for all structural members. To apply wood to wood connections for modern architecture, the mechanical behavior of different types of wood to wood connections should be known.
For wood to wood connections, the friction and bearing stresses from the part of wood member contacting the other part of member are the main resistance against external loads. In other words, the mechanical behavior of the connections was solely dependent upon the properties of materials. Previous studies showed that material properties and failure behaviors associated with structure of the materials were critical to predict strengths of the materials (Jeong and Hindman[1] and Jeong et al.,[2]).

The sagae connection "사개맞춤" as a type of wood to wood connection is assembled with two crossing beams and one column. The tenon and mortise from crossing beams and column

*1 Received on August 22, 2013; accepted on September 24, 2013

*2 Department of Wood Science and Engineering, Chonnam National University, 77 Yongbongro Bukgu, Gwangju 500-757, Korea

*3 Korea Forest Research Institute, 57 Hoegiro, Dongdaemun-gu, Seoul 130-712, Korea

${ }^{\dagger}$ Corresponding author : Gi Young Jeong (e-mail: gjeong1@jnu.ac.kr) 


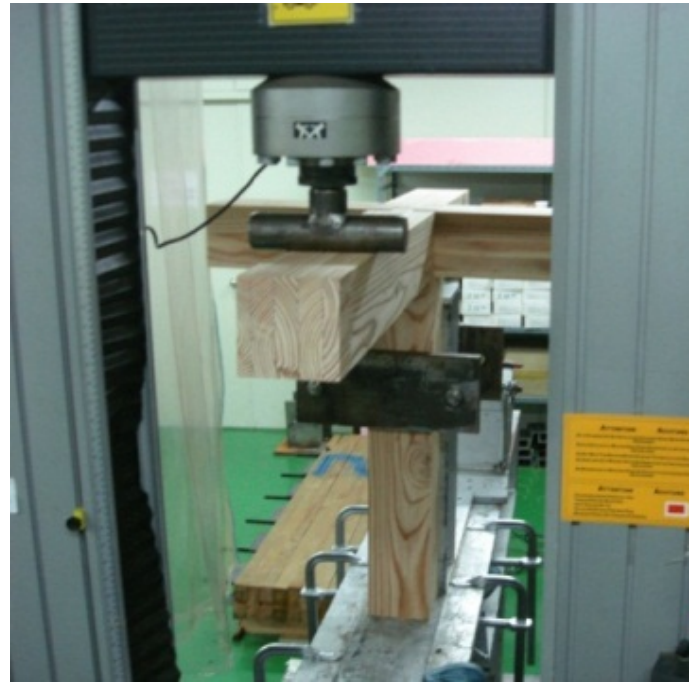

Fig. 1. Experimental test set up for moment carrying capacity of the sagae connection.

could have different geometric factors. However, it is time consuming and costly to investigate the effects of different geometries on the mechanical behavior of the connections. The FEM is an useful tool to simulate the effects of different geometries of mechanical behavior of the connections (Mose and Prion[3], Guan et al.[4], Guan et al.[5], Sangree and Schafer[6], Tannert et al.[7], Tannert et al.[8], Jeong et al.[9]). Once the results of a finite element model were validated by the experimental results, models with different geometries could be explored to analyze its effects on the mechanical behavior of the connections.

The goal of this study is to analyze the effect of geometry on the moment carrying capacity of the sagae connection using the FEM. Different neck widths, mortise depths from beams, and different tenon widths from the column were evaluated to maximize the strength of the sagae connection.

\section{MATERIALS and METHODS}

Fig. 1 shows the sagae connection consisted of two beams and one column. All members for the sagae connection were assembled with glulam materials made of red pine (Larix kaempferi Carr.). The tenon and mortise from the beams and column were processed by a computer numerical controlled (CNC) cutting machine. The beam dimension was $120 \mathrm{~mm}$ by $120 \mathrm{~mm}$ by $700 \mathrm{~mm}$. The crossing beam dimension was $120 \mathrm{~mm}$ by $120 \mathrm{~mm}$ by $520 \mathrm{~mm}$. The column dimension was $120 \mathrm{~mm}$ by $120 \mathrm{~mm}$ by 700 $\mathrm{mm}$. A total of six sagae connections was tested to measure the moment carrying capacity using an universal testing machine (Instron 5585) equipped with a $50 \mathrm{kN}$ load cell. Load was applied at the bottom beam $500 \mathrm{~mm}$ apart from the center of the joint (Fig. 1). Loading rate was $1 \mathrm{~mm} / \mathrm{min}$. The column was supported by the metal fixture tight with two metal plates. The geometric variables of the sagae connection are described in Fig. 2. Different geometries of tenon and mortise from the beams and column for the sagae connections were manufactured using a pre-cut machine. The tested samples of the neck width (a) of the top and bottom beams were $40 \mathrm{~mm}$, the mortise depths $\left(b_{1}\right.$ and $\left.b_{2}\right)$ of the two beams were $60 \mathrm{~mm}$, and the tenon width (c) of the column was $40 \mathrm{~mm}$.

\subsection{Constructing the sagae Connection Using the Finite Element Method}

Three dimensional sagae connections were constructed using ANSYS v11 (ANSYS, inc. USA). Solid 185 element was used to construct three members of the sagae connection. Contact elements were used for the places where the tenon and mortise from different members were met. Friction coefficient of 0.6 was used for the contact element. Input material properties in- 

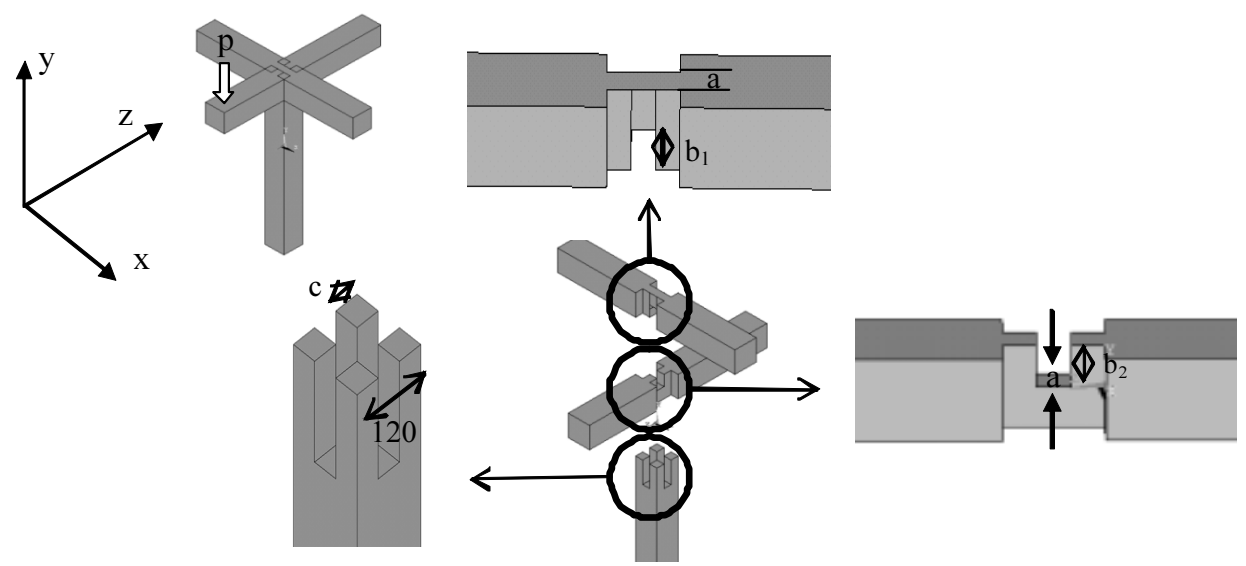

Fig. 2. Geometry variables of the sagae connection.

cluding 11.3 GPa for longitudinal elastic modulus, 0.44 GPa for transverse elastic modulus, $0.7 \mathrm{GPa}$ for shear modulus, and 0.038 for minor Poisson's ratio were applied to represent the mechanical behavior of the glulam used for the sagae connections. Longitudinal and transverse elastic moduli were determined from tension tests, shear modulus and Poisson's ratio were assumed based on elastic ratios (Bodig and Goodman[10]). Shear strength of $12.09 \mathrm{MPa}$ and longitudinal strength of $79.70 \mathrm{MPa}$ were determined from shear block and tension tests, respectively (Hwang et al.[11]). The average elastic modulus and strength values were used for the FEM.

Experimental results showed that brittle failures occurred with a little plastic behavior. From the slope of stress and load relationship, the stress components could be summarized as a function of load and values of slope for the stress components (Jeong and Hindman[1]). The strength of the sagae connection could be predicted using the linear load-stress relationship (Jeong and Hindman [1]). Based on the experimental and simulation results, the critical failure of the sagae connections was governed by the shear and tension parallel to the grain stresses at the reentrant corner of the bottom beam. The failure criterion for structures should reflect failure behavior of the materials (Jeong and Hindman [1]). Therefore, practical failure criteria for the sagae connections could be defined as below.

$$
\frac{\sigma_{z}}{T}+\frac{\tau_{y z}}{S}-1 \leq 0[1]
$$

Where,

$\sigma_{z}$ : the tension parallel to the grain stress from the bottom beam

$\tau_{\mathrm{yz}}$ : shear stress from the bottom beam

$\mathrm{T}$ : the tension parallel to the grain strength

$S$ : the shear strength

Stress values $\left(\sigma_{z}, \tau_{y z}\right)$ associated with the geometric factors from the sagae connections were evaluated from the FEM and strength indexes ( $T, S)$ were obtained from the experimental tests. With the stress values from the FEM and strength indexes from experimental tests, failure loads from the sagae connections associated with different geometric variables were predicted using Equation (1).

To validate the FEM, the average moment 
Table 1. Geometric variables of the mortise and tenon used for the sagae connection

\begin{tabular}{cccc}
\hline $\mathrm{a}(\mathrm{mm})$ & $\mathrm{c}(\mathrm{mm})$ & $\mathrm{b}_{1}(\mathrm{~mm})$ & $\mathrm{b}_{2}(\mathrm{~mm})$ \\
\hline 20 & 50 & 80 & 40 \\
34 & 43 & 64 & 56 \\
37 & 41.5 & 62 & 58 \\
40 & 40 & 60 & 60 \\
43 & 38.5 & 58 & 62 \\
46 & 37 & 56 & 64 \\
49 & 35.5 & 54 & 66 \\
60 & 40 & 52 & 68 \\
& & 40 & 80 \\
\hline
\end{tabular}

carrying capacities of the sagae connections obtained from the experimental test and the FEM were compared. After the validation, different combinations of geometric variables were used to investigate the geometric effects on the stress distribution of the sagae connections. The neck width (a) of the two beams determined the tenon width of the column, whereas the mortise depth $\left(b_{1}\right.$ and $\left.b_{2}\right)$ of the two beams wereindependent from the neck width and the tenon width. The mortise depths of the top beam $\left(b_{1}\right)$ have an inverse relationship with the mortise depths of the bottom beam $\left(b_{2}\right)$.

Table 1 shows different geometric variables used for the analysis. It should be noted that the listed geometric variables were not exact combinations of geometric variables used for the FEM models. Although more combinations of geometric variables than the combinations listed in Table 1 were available, the critical stress distributions associated with the listed geometric variables indicated that the current geometric variables covered the lowest stress distribution of the sagae connection. Different neck widths from Table 1 were used to analyze the stress distributions at the mortise depths $b_{1}$ and $b_{2}$ of $60 \mathrm{~mm}$. After finding the neck width that produced the lowest stress distributions, different mortise depths of the two beams were investigated. Different mortise depth $b_{1}$ and $b_{2}$ from Table 1 were used to analyze the stress distributions at the neck width of $40 \mathrm{~mm}$. From the FEM, the optimized geometric variables for the sagae connections in terms of moment carrying capacity were found.

\section{RESULTS and DISCUSSION}

From the experimental test, the sagae connections shows a brittle failure behavior. The bottom beam was lowered by applied load (Fig. 2). However, friction and bearing forces against other members become dominated and resisted from sliding the bottom beam until failure occurred. Although failure of the connection could not be observed from the surface of the assembled connection, it can be suspected that the failure occurred the inside of the joint area. The average of moment carrying capacity of the sagae connections was found to be $1.709 \mathrm{kN} \cdot \mathrm{m}$.

Although the FEM simulations showed a similar mechanical behavior of the connection observed from experimental test, it could not show any particular stress distribution from the integrated sagae connections. Therefore, critical stress must occur inside of the connections. To analyze the stress distribution of the inside of the connection, each member was disassembled using the FEM.

\subsection{Comparison Between Stress Dis- tributions and Failure Modes from the sagae Connection}

Fig. 3 shows the different stress distributions from simulation with the global coordinate for each member. The tenon width of $40 \mathrm{~mm}$ and 
Predicting Moment Carrying Capacity of the "sagae" Connection Using the Finite Element Method

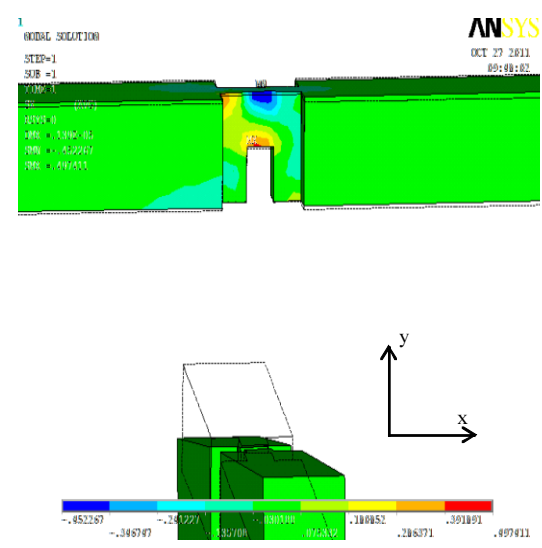

a) Stress $x$ distribution

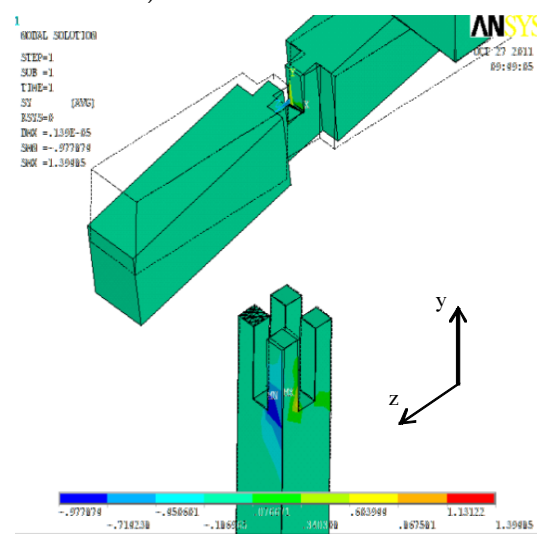

c) Stress y distribution

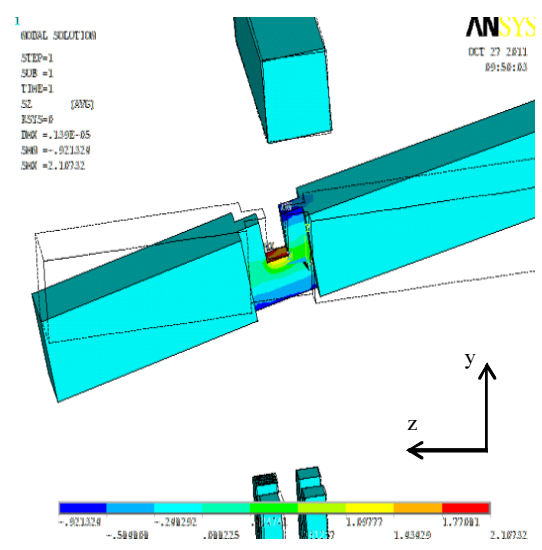

b) Stress $\mathrm{z}$ distribution
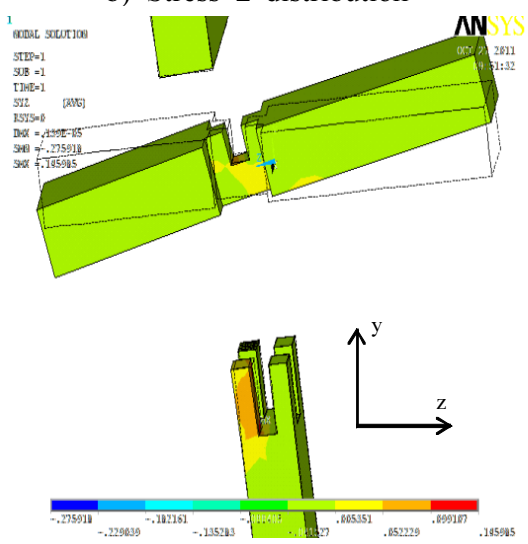

d) Shear yz distribution

Fig. 3. Stress distributions of members in the sagae connection.

mortise depth of $60 \mathrm{~mm}$ from two beams were used for the sagae connections to analyze the stress distribution. The unit for the stress is $\mathrm{Pa}$. Fig. 3a shows stress $x$ distribution of the top beam from the sagae connections. For the top beam, the $\mathrm{x}$-axis is parallel to the fiber direction and the depth of the beam is the y-axis, perpendicular to the fiber direction. The top beam rotated slightly by the applied load at the bottom beam. Compression stress value in the $\mathrm{x}$ direction occurred at the upper part of the mortise and positive tension stress occurred at the lower part of the mortise of the top beam. However, the magnitude of the stress $x$ was found to be much lower than the stress $y$ and stress $\mathrm{z}$ values occurred from other members. Considering the strength value of the material in the longitudinal direction, the stress $\mathrm{x}$ carrying capacity was proportionally higher than other normalized stress values associated with the corresponding strength values.

Fig. $3 b$ shows the stress $\mathrm{z}$ distribution of the bottom beam from the sagae connections. For the bottom beam, the z-axis is parallel to the fiber direction and the depth of the beam is y-axis, perpendicular to the fiber direction. While 


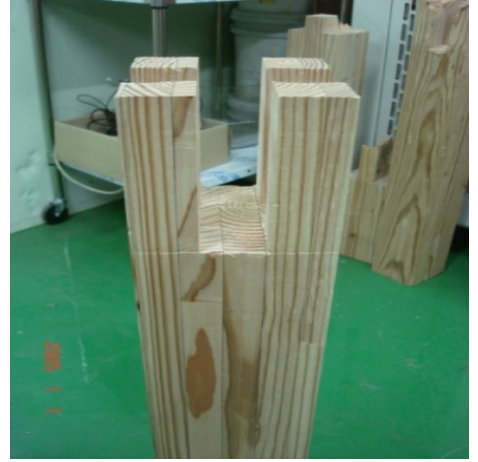

a) Compression in the column

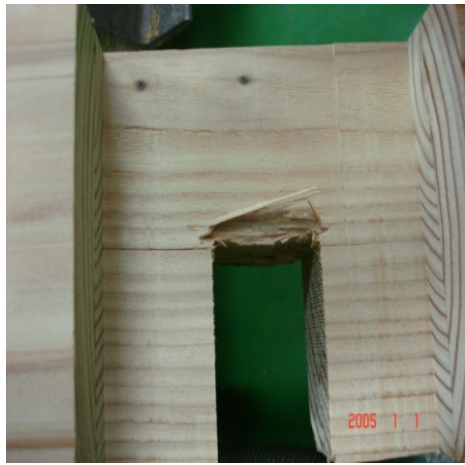

b) Tension in the top beam

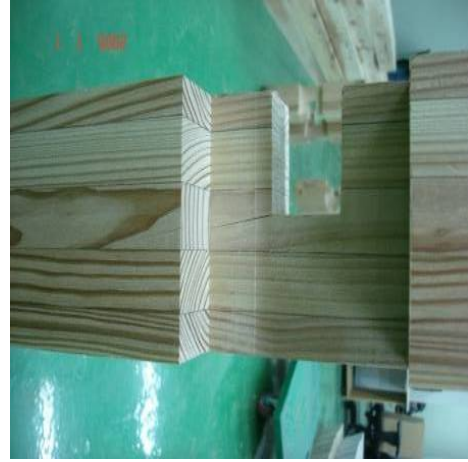

c) Tension and shear at the bottom beam

Fig. 4. Mechanical behavior of the sagae connection after failure.

the column and top beam showed a little movement by the applied load, the bottom beam was displaced noticeably by the applied load. The bottom beam was mainly lowered to the direction of loading because the friction and bearing from each member held the movement against twisting. The tension stress in the longitudinal direction occurred at the reentrant corner due to the fact that bearing strength of the tenon from the column and the back side of the mortise of the top beam held the forward movement of the latter half of the bottom beam.

Fig. 3c shows stress y distribution of the column from the sagae connections. For the column, the length-wise direction is the y-axis, parallel to the fiber direction and the width of the column is the z-axis. As it can be seen from Fig. 3c, the displacement of the column was relatively small compared to the displacement of the bottom beam. The maximum and minimum stress $y$ occurred at the tenon of the column. Compression stress occurred at the left side of the tenon where bending load applied, whereas tension stress occurred at the right side of tenon. The highest shear stress yz occurred at the tenon of the column. The combination of the two stresses associated with the correspond strength were not found to be the highest value compared to the combination of the two stresses occurred at the reentrant corner of the bottom beam.

Fig. $3 \mathrm{~d}$ shows the shear stress yz distribution of the bottom beam and column. The highest shear stress occurred at the tenon of the column. Considering the other stress values, the normalized shear stress yz given the shear strength of the material was not found to be the most critical to lead the failure of the connections.

Although different stress values occurred from each member of the sagae connection, the critical stresses occurred at the reentrant corner of the mortise from the bottom beam (Fig. 3b). The combination of stress $\mathrm{z}$ and shear stress $\mathrm{yz}$ at the reentrant corner of the mortise from the bottom beam was highest compared with any combination values from the other part of different members in the sagae connections.

Fig. 4 shows the members after failure of the sagae connections occurred. The tenon of the column showed the compression mark due to the bottom beam moving against the bottom of the tenon of the column (Fig. 4a). However, it was not critical enough to lead the failure. The mortise of the top beam cracked along the reen- 


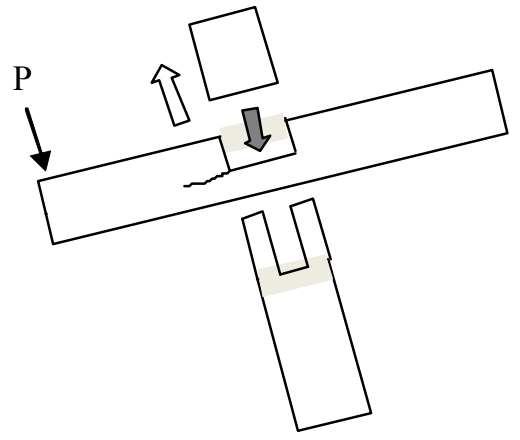

(a)

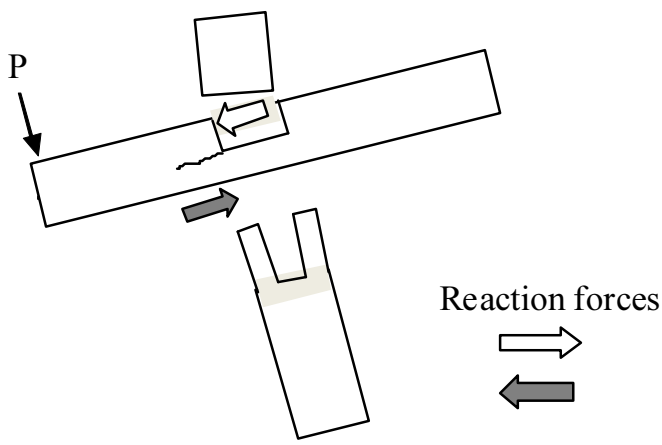

(b)

Fig. 5. Analogy of the failure behaviors of the reentrant corner of the bottom beam from fracture mechanics, a) mode I - opening mode, b) mode II - sliding mode.

trant corner (Fig. 4b). The compression stress at the back side of the mortise of the top beam was induced by the movement of the bottom beam under bending, whereas the tension stress at the front side of the mortise of the top beam occurred due to the tenon of the column, which held the position of the connections. However, the crack of the top beam could not result in the failure of the connection because the rest of the mortise of the beam still can hold the load. Fig. $4 \mathrm{c}$ shows the crack at the reentrant corner of the bottom beam, which resulted in the failure of the connection because the crack at the position significantly reduced the material that could hold load. Fig. 5 shows that the failure mode of the reentrant corner of the bottom beam was similar to the mixed failure mode I and mode II from fracture mechanics. Applied load at the bottom beam lowered the bottom beam while the top beam and the column held the mortise of the bottom beam, which created the mode I behavior (Fig. 5a). The rotation of the top beam was smaller than the bottom beam. The lower part of the shoulder of the top beam pushed the lower part of the left side of the mortise of the bottom beam, which created the mode II behavior (Fig. 5b).

Considering the failure behavior of the sagae connections from the experimental test (Fig. $4 c$ ), the critical stress values were the combination of stress $\mathrm{z}$ and shear stress yz occurred at the reentrant corner of the bottom beam. Therefore, the moment loading capacity of the sagae connections could be determined by the geometric variables of the bottom beam. The moment value of $50.4 \mathrm{~N} \cdot \mathrm{m}$ was applied in the bottom beam. The shear stress value of 0.0548 $\mathrm{N} / \mathrm{m}^{2}$ and stress $Z$ value of $2.07 \mathrm{~N} / \mathrm{m}^{2}$ were found. Since the connection showed a linear load-stress relationship, the maximum moment carrying capacity of the connection could be estimated by multiplying positive real number with the stress values until the combination of stress and strength ratios from Equation 1 was minimized to zero. The maximum moment carrying capacity of the sagae connection was computed to be $1.652 \mathrm{kN} \cdot \mathrm{m}$. The difference of average maximum moment carrying capacity of the sagae connections from the experimental tests and the FEM was $3.33 \%$. Considering the variability of the material properties of the glulam used for the sagae connections, the pre- 
Gi Young Jeong, Moon-Jae Park, Joo-Saeng Park, and Sang-Joon Lee

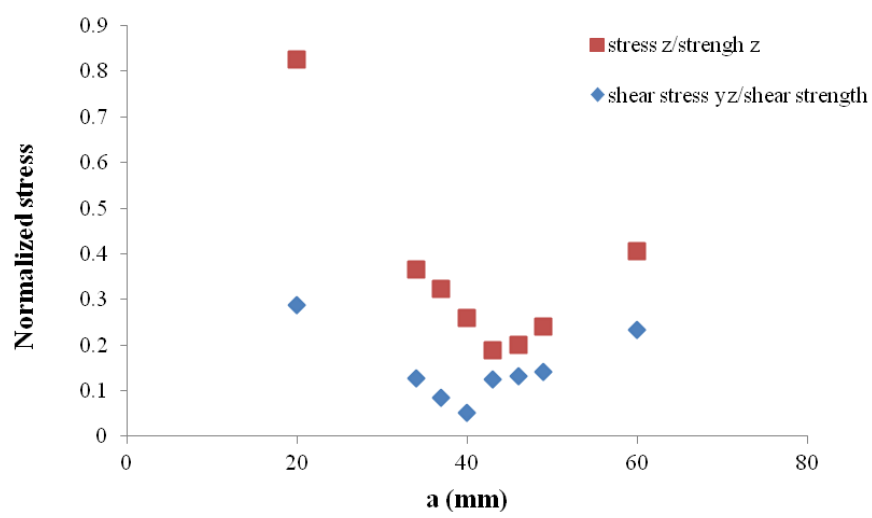

a) Change of normalized stress $\mathrm{z}$ and shear stress associated with neck width (a)

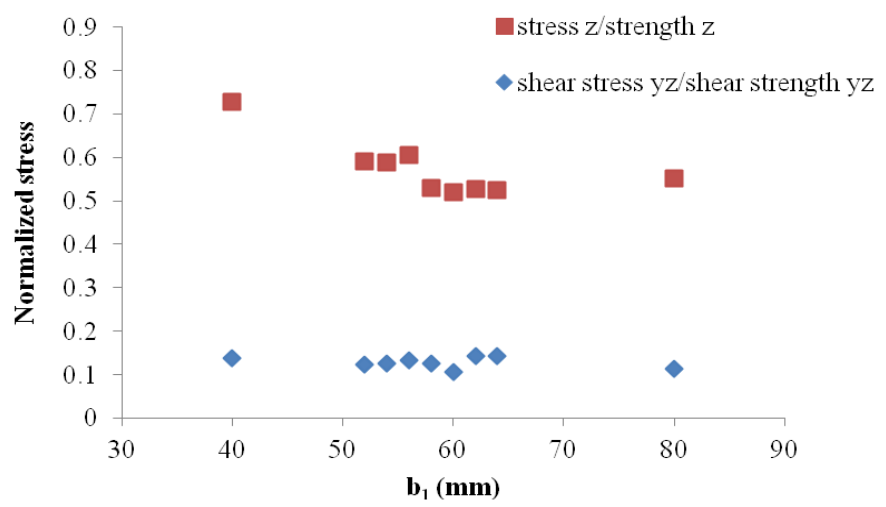

b) Change of normalized stress $\mathrm{z}$ and shear stress associated with the mortise depth $\left(\mathrm{b}_{1}\right)$

Fig. 6. Change of stress values from sagae connection associated with geometric variables.

dicted failure load of the sagae connections from the FEM was reasonable.

\subsection{Changes of Tension Parallel to the Grain Stress and Shear Stress of the Bottom Beam Associated with Geometric Variables in the Sagae Connection}

Fig. 6 shows the change of stress distribution associated with geometric variables of the bottom beam in the sagae connections. The normalized critical stress values from Equation 1 were analyzed. The normalized stress $\mathrm{z}$ values divided by strength $\mathrm{z}$ and the normalized shear stress yz divided by shear strength yz were presented. Fig. 6a shows the normalized stress $\mathrm{z}$ and shear stress yz values with change of the neck width. It is interesting to note that the normalized stress $\mathrm{z}$ value increased after the neck width of $43 \mathrm{~mm}$. The increment of the neck width of the bottom beam reduced the dimension of the tenon of the column, which resulted in the decrement of the stress partitioning of the tenon of the column. Most of the stress $\mathrm{z}$ transferred to the mortise of the bottom beam 
and concentrated at the reentrant corner, which resulted in lowering the loading carrying capacity of the bottom beam.

Although the lowest normalized stress $\mathrm{z}$ was found when the neck width was $43 \mathrm{~mm}$, the lowest normalized shear stress yz was found when the neck width was $40 \mathrm{~mm}$. Minimizing the combination stress values from Equation 1 was found when the neck width was $40 \mathrm{~mm}$. Fig. $6 \mathrm{~b}$ shows the normalized stress $\mathrm{z}$ and shear stress yz values with change of the mortise depth from the top beam. While the normalized stress $\mathrm{z}$ decreased with increment of the mortise depth, the shear stress did not have a significant change with the increment of the mortise depth. The combination of the normalized stress $\mathrm{z}$ and shear stress was found to be the lowest when the mortise depth was $60 \mathrm{~mm}$. It is interesting that larger than mortise height of $60 \mathrm{~mm}$ from the bottom beam did not reduce the stress $\mathrm{z}$ at the reentrant corner of the bottom beam. In fact, it was observed that the normalized stress $\mathrm{z}$ value from mortise height of $80 \mathrm{~mm}$ from the bottom beam was higher than that from the mortise height of $60 \mathrm{~mm}$. It can be interpreted that the dominant failure behavior of the bottom beam with a larger height of $60 \mathrm{~mm}$ occurred by the mixed mode I and mode II associated with a little partitioning of stress transferring from the top beam.

\section{CONCLUSIONS}

The effects of the geometry variables on moment carrying capacity of the $120 \mathrm{~mm}$ by 120 $\mathrm{mm}$ sagae connections assembled with glulam materials made of red pine were investigated. Failure behavior of the sagae connections from the experimental test was dominated by the crack at the reentrant corner of the bottom beam. The FEM model showed that the critical failure occurred at the reentrant corner happen by the mixed stress values of stress $\mathrm{z}$ and shear stress yz. Compared to the estimated moment carrying capacity from experimental test, the difference of the predicted moment carrying capacity from the FEM was found to be $3.33 \%$. The highest moment carrying capacity of the sagae connections was found to be when the mortise of the bottom beam was $60 \mathrm{~mm}$ and the neck width of the mortise was $40 \mathrm{~mm}$. From the results of the FEM and experimental test, The FEM can be used for predicting the moment carrying capacity of the sagae connections.

\section{ACKNOWLEDGEMENT}

This study was financially supported by Chonnam National University and Korea Forest Research Institute, 2013.

\section{REFERENCES}

1. Jeong, G. Y. and D. P. Hindman. 2009. Ultimate tensile strength of loblolly pine strands using stochastic finite element method. J mater Sci. 44(14): 3824 832.

2. Jeong G. Y., D. P. Hindman, A. Zink-Sharp. 2010. Orthotropic properties of loblolly pine (Pinus taeda) strands. J Mater Sci. 45(21): 5820 830.

3. Mose D. M. and H. G. L. Prion. 2004. Stress and failure analysis of wood composites: a new model. Compostes: Part B. 35(3): 251 61.

4. Guan Z. W., A. Kitamori, K. Komatsu. 2008. Experimental study and finite element modeling of Japanese "Nuki" joints - part one: initial stress states subjected to different wedge configurations. Engineering Structure 30(7): 2032 040.

5. Guan Z. W., A. Kitamori, K. Komatsu. 2008. Experimental study and finite element modeling of Japanese "Nuki" joints - part two: racking resistance subjected to different wedge configurations. Engineering Structure 30(7): 2041 049.

6. Sangree R. H. and B. W. Schafer. 2009. Experimental and numerical analysis of a halved and 
Gi Young Jeong, Moon-Jae Park, Joo-Saeng Park, and Sang-Joon Lee

tabled traditional timber scarf joint. Construction and Building Materials 23(2): 615 24.

7. Tannert T., F. Lam, and T. Vallée. 2010. Strength prediction for rounded dovetail connections considering size effects. J Engineering Mechanics 136(3): 358 66.

8. Tannert T., F. Lam, and T. Vallée. 2011. Structural performance of rounded dovetail connections: experimental and numerical investigations. Eur J. Wood Prod 69(3): 471 82.

9. Jeong G. Y., M. J. Park, J. S. Park. and K. H. Hwang. 2012. Predicting load carrying capacity of dovetail connection using the stochastic finite element method. Wood Fiber Sci. 44(4): 430 39.

10. Bodig J. and J. R. Goodman. 1973. Prediction of elastic parameters for wood. Wood Sci. 5(4): 249 64.

11. Hwang K. H. and J. S. Park. 2008. Estimation of moment resisting property for pin connection using shear strength of small glulam specimens. Mokchae Konghak. 36(4): 58-5. In Korean with summary in English. 